\title{
Composición de la flora arbórea y arbustiva en cinco áreas verdes del Centro Histórico del Cusco
}

\section{Composition of the arboreal and shrub flora in five green areas of the Historic Center of Cusco}

$\underline{\text { Artículo }}$

\section{Rafael De La Colina Lozada ${ }^{1}$, Julissa Evelin Secca Blanco²}

1. Doctorado en Medio Ambiente y Desarrollo Sostenible, Universidad Andina del Cusco, Cusco, Perú. rafitocolina@gmail.com, https://orcid.org/0000-0003-1349-0128

2. Universidad Nacional de San Antonio Abad del Cusco, Cusco, Perú. biojulis@gmail.com, https://orcid.org/0000-0002-9173-459X

\section{Págs.: 132 - 141}

Recibido: $7 / 9 / 2020$

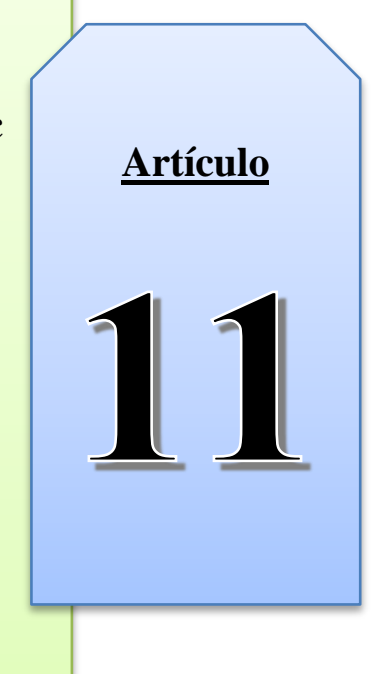




\begin{abstract}
The green areas of the Historic Center of Cusco, present a high diversity of flora species. Due to the aforementioned, the objective of the study is to determine the composition of the arboreal and shrub flora in five green areas of the Historic Center. To this end, the evaluation was carried out in five places, Plazoleta de Santa Ana, Plaza San Francisco, Plaza Regocijo, Plaza de Armas, and Parque de la Madre, through direct observation, determination of the species through the help of flora guides, and the experience of the authors. A total of 170 individuals were recorded, belonging to 49 species of which 26 are trees and 23 shrubs. As for the place where there was more diversity, the Plaza San Francisco presented 42 species and 95 individuals. Regarding the trees, 88 individuals were registered, representing $51.76 \%$ of the total registered. There are 82 shrub species registered, representing 48.24\%. Regarding the origin of the species, 37 are native, which represents $75.51 \%$, and the introduced ones are 12 represents $24.49 \%$, this difference is notorious because due to the climatic conditions of Cusco, the growth of arboreal and shrub species introduced in little successful. This work is the first to be done comparing various green areas. Likewise, the use of native tree and shrub species is more prevalent as ornamental plants.
\end{abstract}

Key words: Historic Center, Green areas, Trees, Shrubs, Native, Introduced

\title{
Introducción
}

Superficie de área verde por habitante, es la superficie de área verde urbana por habitante que cuenta las ciudades. Las áreas verdes son espacios públicos compuestos con vegetación, sobre todo, pastos, árboles y algunos arbustos. Según la Organización Mundial de la Salud (OMS) señala que la existencia de espacios libres es una necesidad cada vez más urgentes en el plano regional, nacional e incluso internacional; la expansión sin precedentes de las grandes concentraciones urbanas ha hecho que la disponibilidad de espacios libres sea una necesidad imperiosa, Así mismo recomienda que las ciudades deberán cumplir mínimamente con $9 \mathrm{~m}^{2}$ de áreas verdes por habitante. La protección y conservación de los recursos naturales, y de los espacios libres suelen exigir la intervención del Estado. Las zonas verdes, los paseos arbolados y los espacios libres de todo tipo enlazan lógicamente el hogar, la vecindad y la aglomeración urbana con el campo circundante, y deberán ser una de los rasgos más visibles del mapa metropolitano y de la ciudad misma. Las ciudades en expansión requieren del desarrollo y mantenimiento de áreas verdes a fin de no sólo proveer áreas de recreación a los habitantes del lugar, sino para contribuir a captar el CO2. (MINAM 2016).

Los espacios verdes son esenciales para el desarrollo urbano sustentable. Los parques, las plazas junto con los arboles urbanos no solo definen en gran medida la identidad y el atractivo de las ciudades; estos espacios abiertos contribuyen además a la calidad de vida de sus habitantes. En el Centro Histórico del Cusco existe aproximadamente 1.33 $\mathrm{m}^{2}$ de área verde neta pública por habitante, no obstante que la Organización Mundial 
de la Salud (OMS) establece un rango óptimo por habitante de 10 a $15 \mathrm{~m}^{2} / \mathrm{hab}$. En relación al área total del Centro Histórico del Cusco (CHC), solamente el 10\% está destinado a áreas verdes, ubicadas en plazas, jardines interiores - exteriores, pequeños huertos, bermas y óvalos. (MPC 2018).

\section{Metodología}

El área de estudio es en la ciudad del Cusco, que se ubica en los Andes Centrales, al sudeste del Perú, entre los $12^{\circ} 71^{\prime} 11^{\prime \prime}$ de latitud sur y $72^{\circ} 00^{\prime} 49^{\prime \prime}$ de longitud oeste a partir del meridiano de Greenwich, a una altura promedio de 3,300 msnm. El CHC se ubica en la parte noroeste del valle del Cusco, en la zona 19 entre las coordenadas 177 534 abscisa este y 8503762 ordenada norte del sistema de proyección Universal Transversal Mercator (UTM). El CHC, espacio donde habitan casi 74 mil habitantes está compuesto por 598 manzanas, para su análisis e intervención se ha mantenido el criterio de establecer dos áreas de estructuración AE-I (Centro Histórico) y AE-II (Área Circundante de Protección). En el AE-I existen 411 inmuebles con características monumentales y patrimoniales y 49 ambientes urbanos monumentales. El AE-II si bien no cuenta con la misma especificidad de catalogación en sus inmuebles, alberga también algunas edificaciones y ambientes urbanos con características monumentales y patrimoniales. Las áreas verdes usadas para el estudio fueron la Plazoleta de Santa Ana, Plaza San Francisco, Plaza Regocijo, Plaza de Armas, y el Parque de la Madre. (Figuras 1 y 2)

El estudio se realizó la última semana del mes de agosto del 2020, se tomaron todas las medidas de seguridad, como el uso de mascarillas, caretas y alcohol al 70\%, se recorrió por las áreas verdes donde mediante observación directa se registraron todas las especies de árboles y arbustos presentes en dichas áreas, mediante el uso de una libreta de campo, lápiz y se realizó el registro fotográfico de las especies, la identificación se realizó a nivel de familia, especie, nombre común, número de individuos, así como si eran especies nativas o introducidas.

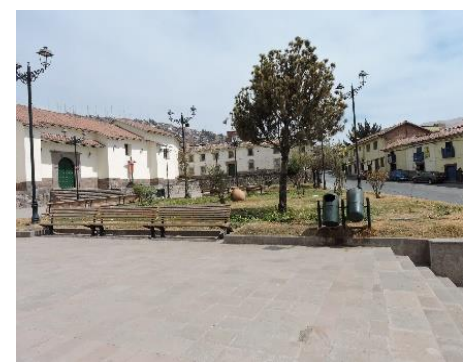

Plazoleta de Santa Ana

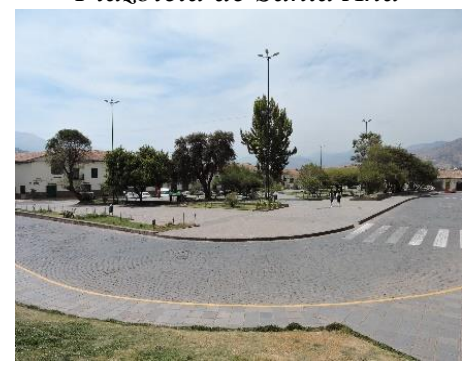

Plaza San Francisco

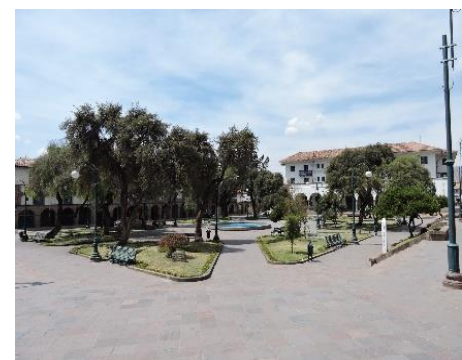

Plaza Regocijo

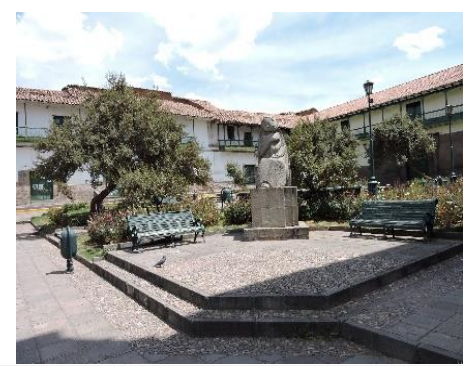

Parque de la Madre

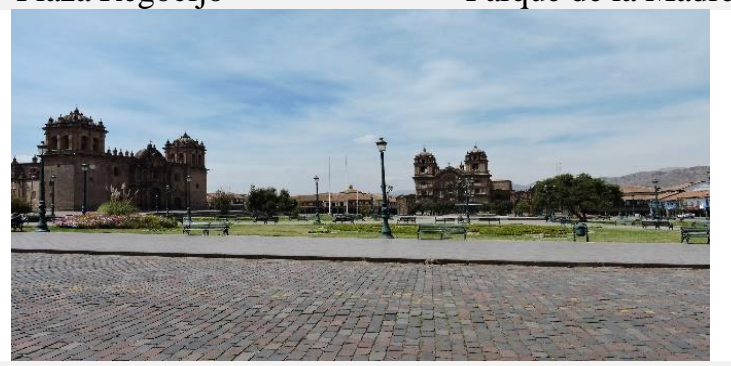

Plaza de Armas 
Figura 1. Áreas verdes evaluadas en el Centro Histórico del Cusco

Para la identificación correcta de las especies de árboles y arbustos se utilizó la Guía de Arboles de Lima, Servicio de Parques de Lima- SERPAR (2011), diversas fuentes electrónicas referidas a flora, así como la experiencia de los autores en trabajos botánicos. Para determinar la denominación correcta de los nombres científicos se utilizó la base de datos de la página electrónica de Trópicos (2020).

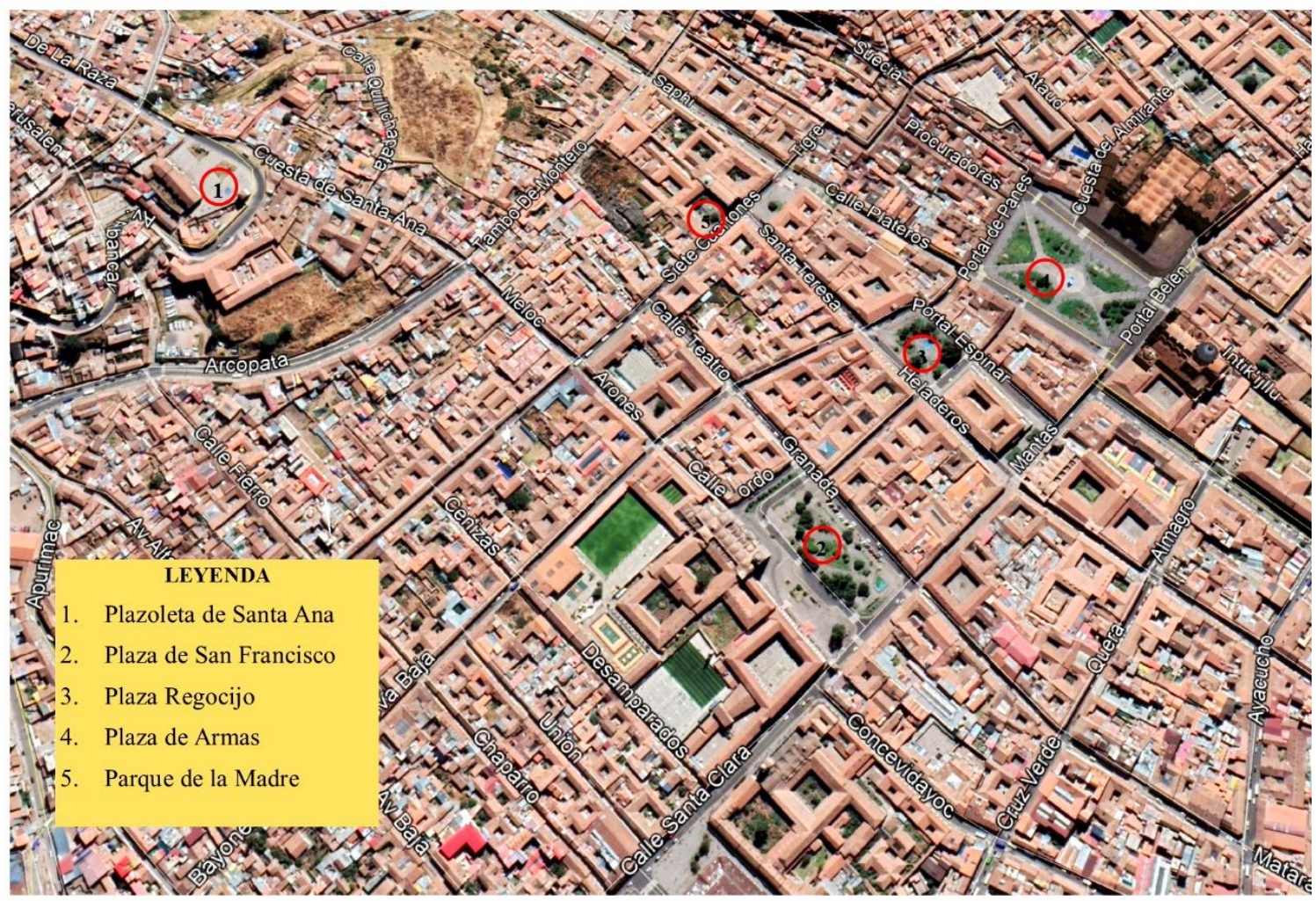

Figura 2. Ubicación de las áreas verdes en el Centro Histórico del Cusco. (Google inc., 2020) Recuperado de: Google earth

\section{Resultados y Discusión}

En el área de estudio se logró registrar un total 170 individuos, pertenecientes a 49 especies de las cuales 26 son árboles y 23 arbustos. La Plaza San Francisco en la que presenta el mayor número de especies (42) e individuos (95), seguida de la Plazoleta de Santa Ana, después esta la Plaza Regocijo, a continuación la Plaza de Armas y finalmente el Parque de la Madre presenta el menor número de especies (1) e individuos (9). (Figura 3). 


\section{GUACAMAYA}

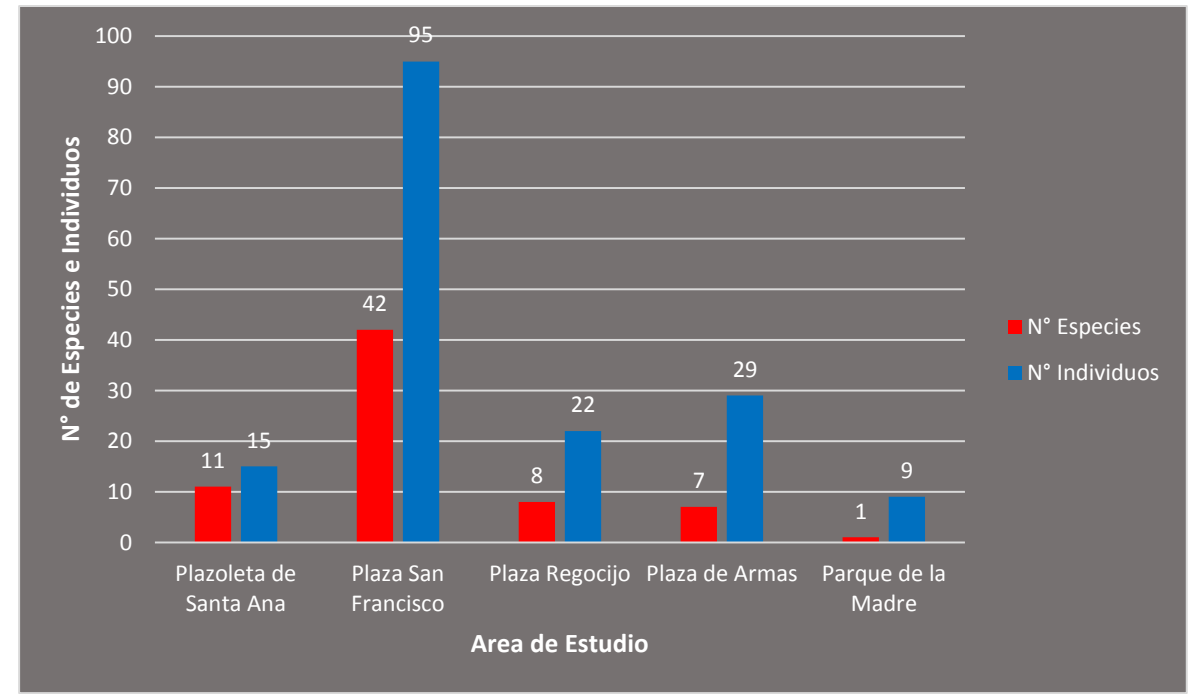

Figura 3. Número de especies e individuos registrados en el área de estudio

En cuanto a los árboles se lograron registrar 88 individuos que representan el 51.76\% del total registrado, la Plaza de San Francisco es la que presenta en mayor número de árboles 44 que representa el 50\% del total de árboles registrados, y el Parque de la Madre presenta el menor número 9 que representa el 10.23\% del total registrado. (Tabla $1)$.

Los individuos de especies arbustivas registradas son 82 , que representan el $48.24 \%$ del total registrado, la Plaza de San Francisco es la que presenta en mayor número de arbustos 51 que representa el 66.20\% del total de arbustos registrados, y el Parque de la Madre no presenta ningún arbusto. (Tabla 2). (Figura 4).

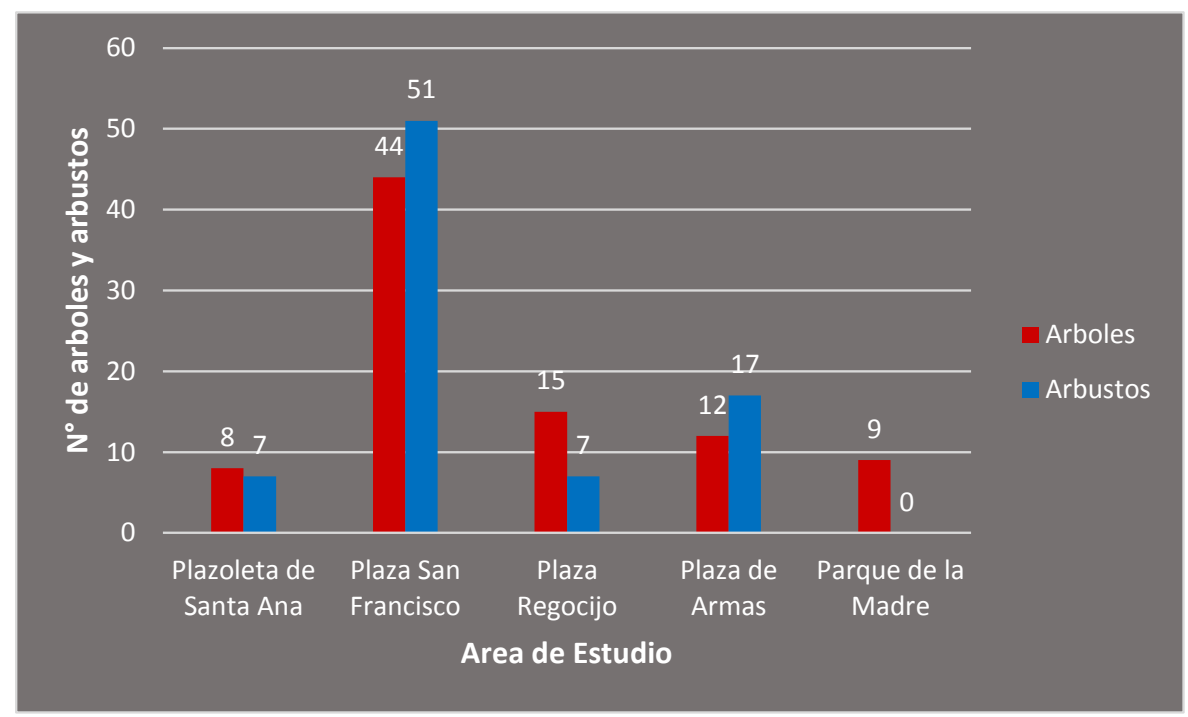

Figura 4. Número de árboles y arbustos registrados en el área de estudio 
GUACAMAYA

En cuanto al origen de las especies 37 son nativas lo que representa el 75.51\%, y las especies introducidas son 12 que representa el $24.49 \%$, esta diferencia es notoria debido a que por las condiciones climáticas de Cusco el crecimiento de especies arbóreas y arbustivas introducidas en un poco exitoso, sin embargo por la vistosidad de muchas de estas muchas personas están optando por usarlas sobre todo para ornamentar viviendas, hoteles, calles y avenidas, otro factor es que debido al cambio climático las condiciones climáticas están cambiando lo que contribuye al incremento en el cultivo de especies arbóreas y arbustivas introducidas.

Tabla 1

Especies de Árboles registrados en las áreas verdes

\begin{tabular}{|c|c|c|c|c|}
\hline Familia & Nombre Científico & Nombre Común & Origen & $\mathrm{N}^{\circ}$ Individuos \\
\hline \multirow{2}{*}{ Anacardiaceae } & Schinus molle & molle & Nativa & 5 \\
\hline & Schinus pearcei & china molle & Nativa & 2 \\
\hline Araucariaceae & Araucaria excelsa & araucaria & Introducida & 1 \\
\hline Arecaceae & Phoenix dactylifera & palmera & Introducida & 2 \\
\hline Betulaceae & Alnus acuminata & aliso & Nativa & 4 \\
\hline \multirow{2}{*}{ Bignoniaceae } & Delostoma integrifolia & huaruma & Nativa & 1 \\
\hline & Tecoma sambucifolia & huaranhuay & Nativa & 2 \\
\hline Caprifoliaceae & Sambucus peruviana & sauco & Nativa & 1 \\
\hline Casuarinaceae & Casuarina equisetifolia & pino australiano & Introducida & 1 \\
\hline \multirow{2}{*}{ Fabaceae } & Erythrina falcata & pisonay & Nativa & 1 \\
\hline & Acacia macracantha & huarango & Nativa & 1 \\
\hline Grossulariaceae & Escallonia resinosa & chachacomo & Nativa & 10 \\
\hline Lauraceae & Persea americana & palta & Nativa & 1 \\
\hline Mytaceae & Myrcianthes oreophila & unca & Nativa & 5 \\
\hline Oleaceae & Olea europaea & olivo & Introducida & 1 \\
\hline Pinaceae & Pinus radiata & pino & Introducida & 2 \\
\hline Podocarpaceae & Podocarpus glomeratus & intimpa & Nativa & 5 \\
\hline Proteaceae & Grevillea robusta & grevillea & Introducida & 1 \\
\hline \multirow{6}{*}{ Rosaceae } & Prunus persica & durazno & Introducida & 2 \\
\hline & Polylepis incana & queuña & Nativa & 2 \\
\hline & Polylepis microphylla & ñutu queuña & Nativa & 1 \\
\hline & Polylepis racemosa & queuña & Nativa & 27 \\
\hline & Prunus serotina & capuli & Nativa & 1 \\
\hline & Kageneckia lanceolata & lloqu'e & Nativa & 3 \\
\hline Salicaceae & Salix babylonica & sauce lloron & Introducida & 4 \\
\hline Verbenaceae & Citharexylum herrerae & $\begin{array}{l}\text { huayruro } \\
\text { cusqueño }\end{array}$ & Nativa & 2 \\
\hline
\end{tabular}


Tabla 2

Especies de Arbustos registrados en las áreas verdes

\begin{tabular}{|c|c|c|c|c|}
\hline Familia & Nombre Científico & Nombre Común & Origen & $\mathrm{N}^{\circ}$ Individuos \\
\hline Asparagaceae & Yucca gloriosa & $\begin{array}{l}\text { chamagra del } \\
\text { Perú }\end{array}$ & Introducida & 2 \\
\hline \multirow{3}{*}{ Asteraceae } & Mutisia acuminata & chinchurcuma & Nativa & 1 \\
\hline & Baccharis odorata & tayanka & Nativa & 1 \\
\hline & Baccharis latifolia & ch'illca & Nativa & 2 \\
\hline Berberidaceae & Berberis commutata & q'eswa ch'eqche & Nativa & 1 \\
\hline Euphorbiaceae & Acalypha aronioides & p'ispita & Nativa & 2 \\
\hline \multirow{3}{*}{ Fabaceae } & Spartium junceum & retama & Introducida & 1 \\
\hline & Senna multiglandulosa & mutuy & Nativa & 5 \\
\hline & Caesalpinia spinosa & tara & Nativa & 4 \\
\hline \multirow{2}{*}{ Loganiaceae } & Buddleja coriacea & colle & Nativa & 1 \\
\hline & Buddleja incana & quiswar & Nativa & 1 \\
\hline Malvaceae & Abutilon molle & rata rata & Nativa & 1 \\
\hline \multirow[t]{2}{*}{ Onagraceae } & Fuchsia hybrida & fucsia & Introducida & 1 \\
\hline & Cantua buxifolia & kantu rojo & Nativa & 22 \\
\hline \multirow[t]{2}{*}{ Polemoniaceae } & Cantua pyrifolia & kantu amarillo & Nativa & 11 \\
\hline & Cantua bicolor & kantu rosado & Nativa & 2 \\
\hline Rhamnaceae & Colletia spinosissima & roqu'e & Nativa & 1 \\
\hline \multirow[t]{3}{*}{ Rutaceae } & Swinglea glutinosa & limoncillo & Introducida & 12 \\
\hline & Brugmansia arborea & campanchu & Nativa & 2 \\
\hline & Cestrum conglomeratum & kúsmayllu & Nativa & 5 \\
\hline \multirow[t]{3}{*}{ Solanaceae } & Dunalia ovobata & t'ankar & Nativa & 1 \\
\hline & Solanum maturecalvans & asnaqsach'a & Nativa & 2 \\
\hline & Lycianthes lycioides & t'ankar kiska & Nativa & 1 \\
\hline
\end{tabular}

En general, se observa la presencia de diversas especies de árboles y arbustos, tanto nativos, no nativos y exóticos, que son comúnmente empleados en la arboricultura urbana Pereira (2019. La Plaza San Francisco presenta la mayor diversidad y abundancia de especies de árboles y arbustos nativos, que fueron utilizados desde hace muchos años como lo menciona Quintanilla (2015), existe un predominio de las especies arbóreas utilizadas para la ornamentación de plazas y jardines como lo reporta Paredes (2017), así mismo se observa una prevalencia de las especies nativas arbóreas, las cuales están mejor adaptadas al lugar donde se plantan, y favorecen a la fauna del entorno y necesitan menos cuidados como lo menciona Cevallos (2017). El estudio de las áreas verdes en los espacios urbanos tiene cada vez mayor importancia no sólo para los organismos de gestión sino también para los habitantes Benedetti (2016), debido a la importancia tanto a nivel ambiental como de salud. Sin embargo la expansión de las ciudades promueve el reemplazo de biotas locales (nativas) por especies exóticas, lo que causa una disminución en la diversidad como menciona Santilli (2018). 
Como se ve en la tabla 1 Polylepis racemosa "queuña" es la especie arbórea más abundante en las áreas verdes evaluadas, esto debido a que es una especie nativa y representativa de los andes, Polylepis se encuentra distribuido en 19 departamentos, de los 24 departamentos del Perú como menciona Mendoza (2011), seguida de Escallonia resinosa "chachacomo" que también es una especie nativa muy conocida y apreciada, que está representado en el Perú, en los departamentos de Ancash, Ayacucho, Cajamarca, Cusco, Junín y La Libertad Orihuela (2019).

En cuanto a los arbustos Cantua buxifolia "kantu rojo", es la especie arbustiva más abundante en las áreas verdes evaluadas, esta es una especie nativa muy apreciada como indica Salaverry (2014) Flor nacional del Perú en la época incaica se consagraba al sol o Inti y por eso su amplia difusión, por lo cual es muy utilizada como especies ornamental por lo vistoso de su flores.

Se debe tener en consideración el mantenimiento y la ampliación de las áreas verdes, como la Implementación y protección de áreas verdes en espacios públicos del CHC como se menciona en Municipalidad Provincial del Cusco (2018), así mismo es lamentable observar la presencia de perros callejeros en las áreas verdes del CHC, los cuales dejan sus heces dentro de estas convirtiéndose en un foco de contaminación tanto para la población como para el personal encargado de su mantenimiento el cual muchas veces no es eficiente.

\section{Conclusiones}

La información obtenida en el estudio es la primera que se realiza comparando varias áreas verdes en el CHC.

Se determinó la presencia de especies de árboles y arbustos, tanto nativas como introducidas, siendo las nativas las de mayor prevalencia en las áreas verdes, esto se debe mayormente a que hay un arraigo de parte de la población por el uso en la ornamentación de especies arbóreas y arbustivas nativas, y esto influye en la decisión de las autoridades encargadas de estas actividades.

Las especies nativas Polylepis racemosa "queuña" Escallonia resinosa "chachacomo", y Cantua buxifolia "kantu rojo", son las que mayor presencia tienen en las áreas verdes evaluadas, lo cual debe ser replicado en otras áreas verdes de la provincia del Cusco.

Al considerarse la Plaza San Francisco como un jardín botánico, esta presenta una mayor diversidad de árboles y arbustos y el mantenimiento y el reemplazo de los individuos dañados se hace con mayor frecuencia por dicha denominación. 
De igual manera se debe de realizar el mantenimiento adecuado y su fuese posible la colocación nuevamente de placas con los nombre comunes y científicos de las especies en las áreas verdes del CHC, ya que dichas áreas son utilizadas para la enseñanza de cursos de botánica, ecología o guidismo por parte de universidades e institutos, así como son apreciadas por parte de los turistas que en gran cantidad arriban a la ciudad del Cusco cada año.

\section{Recomendaciones}

Se debe realizar más trabajos referentes a las especies de flora y fauna presentes en la provincia del Cusco.

Se debe incluir en las evaluaciones de flora las especies herbáceas.

Se debe promover el uso de estas áreas verdes para promover el conocimiento de las especies de flora nativa para estudiantes a nivel escolar, técnico, universitario, y para la población en general.

\section{Referencias bibliográficas}

Benedetti, G. M., Duval, V. S., \& Campo, A. M. (2016). Propuesta para el análisis de cobertura del arbolado urbano: Caso de estudio: Pigüé, provincia de Buenos Aires.

Cevallos, A., González, P., Asanza, M., Neill, D., Gutiérrez, D., \& Martínez, L. (2017). Flora de parques y avenidas de Latacunga. UTCiencia" Ciencia y Tecnología al servicio del pueblo", 1(1), 20-31.

Google inc. (2020). Recuperado de: Google earth pro.

Mendoza, Wilfredo, \& Cano, Asunción. (2011). Diversidad del género Polylepis (Rosaceae, Sanguisorbeae) en los Andes peruanos. Revista Peruana de Biología, 18(2), 197-200. Recuperado en 03 de septiembre de 2020, de http://www.scielo.org.pe/scielo.php?script=sci_arttext\&pid=S1727.9332011000 200011\&lng=es\&tlng=es.

Municipalidad Provincial del Cusco. (2018). Actualización del Plan Maestro del Centro Histórico del Cusco 2018-2028. Cusco: Municipalidad Provincial del Cusco.

Orihuela Izaguirre, W. B., \& Gurmendi Porras, C. G. (2019). Valoración económica de la reserva de carbono de Escallonia resinosa (Ruiz \& Pav.) Pers. en San Pedro de Saños y Pucará.

Paredes, E. C. (2017). Composición y características del arbolado del Reparto Hermanos Cruz. Ciencias Forestales y Ambientales, 2(2), 174-180.

Pereira, E., Flores, D., \& Castillo, M. Caracterización de la flora leñosa de los principales espacios verdes urbanos de la parroquia Táriba, municipio Cárdenas, estado Táchira. Venezuela. 
Quintanilla, E. R. (2015). Diagnóstico y evaluación con fines de mejoramiento y rehabilitación para el desarrollo sostenible del jardín etnobotánico K'ayra Cusco (p. 181). Retrieved from http://repositorio.unsaac.edu.pe/handle/UNSAAC/128

Salaverry O, Cabrera J. Florística de algunas plantas medicinales [galería]. Rev Peru Med Exp Salud Pública. 2014;31(1):165-8.

Santilli, Ludovica, Castro, Sergio A., Figueroa, Javier A., Guerrero, Nicole, Ray, Cristian, Romero-Mieres, Mario, Rojas, Gloria, \& Lavandero, Nicolás. (2018). Exotic species predominates in the urban woody flora of central Chile. Gayana. Botánica, 75(2), 568-588. https://dx.doi.org/10.4067/S071766432018000200568 